\title{
Fibromyalgia diagnostic model derived from combination of American College of Rheumatology 1990 and 2011 criteria
}

\author{
Banafsheh Ghavidel-Parsa ${ }^{1}$, Ali Bidari ${ }^{2}$, Asghar Hajiabbasi ${ }^{1}$, Irandokht Shenavar ${ }^{1}$, \\ Babak Ghalehbaghi ${ }^{3}$, and Omid Sanaei ${ }^{4}$ \\ ${ }^{1}$ Rheumatology Research Center, Razi Hospital, School of Medicine, Guilan University of Medical Sciences, Rasht, \\ ${ }^{2}$ Department of Rheumatology, Iran University of Medical Sciences, Tehran, \\ ${ }^{3}$ ENT and Head and Neck Research Center and Department, Iran University of Medical Sciences, Tehran, Iran, \\ ${ }^{4}$ Division of Gastroenterology and Hepatology, Johns Hopkins Medical Institutions, Baltimore, MD, USA
}

Background: We aimed to explore the American College of Rheumatology (ACR) 1990 and 2011 fibromyalgia (FM) classification criteria's items and the components of Fibromyalgia Impact Questionnaire (FIQ) to identify features best discriminating FM features. Finally, we developed a combined FM diagnostic (C-FM) model using the FM's key features.

Methods: The means and frequency on tender points (TPs), ACR 2011 components and FIQ items were calculated in the FM and non-FM (osteoarthritis [OA] and non-OA) patients. Then, two-step multiple logistic regression analysis was performed to order these variables according to their maximal statistical contribution in predicting group membership. Partial correlations assessed their unique contribution, and two-group discriminant analysis provided a classification table. Using receiver operator characteristic analyses, we determined the sensitivity and specificity of the final model.

Results: A total of 172 patients with FM, 75 with OA and 21 with periarthritis or regional pain syndromes were enrolled. Two steps multiple logistic regression analysis identified 8 key features of FM which accounted for $64.8 \%$ of variance associated with FM group membership: lateral epicondyle TP with variance percentages (36.9\%), neck pain (14.5\%), fatigue (4.7\%), insomnia (3\%), upper back pain (2.2\%), shoulder pain (1.5\%), gluteal TP (1.2\%), and FIQ fatigue (0.9\%). The C-FM model demonstrated a 91.4\% correct classification rate, $91.9 \%$ for sensitivity and $91.7 \%$ for specificity.

Conclusions: The C-FM model can accurately detect FM patients among other pain disorders. Re-inclusion of TPs along with saving of FM main symptoms in the C-FM model is a unique feature of this model. (Korean J Pain 2019; 32: 120-8)

Key Words: Chronic pain; Discriminant analysis; Fatigue; Fibromyalgia; Logistic models; Neck pain; Osteoarthritis; Periarthritis; Sensitivity and specificity; Shoulder pain; Sleep initiation and maintenance disorders; Survey and questionnaires.

Received November 7, 2018. Revised March 2, 2019. Accepted March 4, 2019.

Correspondence to: Ali Bidari

Department of Rheumatology, Iran University of Medical Sciences, Hazarat Rasoul Medical Complex, Sattarkhan Ave, Tehran 41448-95655, Iran Tel: +98-91-2327-7847, Fax: +98-21-6652-5327, E-mail: bidari.a@iums.ac.ir

ORCID: https://orcid.org/0000-0002-3583-1838

() This is an open-access article distributed under the terms of the Creative Commons Attribution Non-Commercial License (http:// creativecommons.org/licenses/by-nc/4.0/), which permits unrestricted non-commercial use, distribution, and reproduction in any medium, provided the original work is properly cited.

(c) The Korean Pain Society, 2019 


\section{INTRODUCTION}

Fibromyalgia (FM) is often considered as part of a diverse spectrum of heterogeneous symptoms that lack precise classification [1,2]. Over the last 4 decades, much effort has been made to recognize the best discriminative set of FM diagnostic criteria. However, the FM criteria have undergone numerous revisions since first reported [3]. For a long period of time the mainstay of FM diagnosis was the American College of Rheumatology (ACR) 1990 FM classification criteria which was based on the presence of widespread pain and tender points (TPs) on examination [4]. But the examination of TPs did not address FM symptoms and was hard to perform for most non-rheumatologists [5-7].

The ACR 2010 criteria [6] and its self-reported version, the modified ACR 2011 criteria [7] not only eliminated TPs, but also changed the case definition of FM to an illness characterized by self-reported, multiple painful regions and additional key symptoms, such as problems with fatigue, sleep, cognition, and the extent of somatic symptom reporting. The modification to the ACR 2010 diagnostic criteria was to substitute a count of 3 symptoms (headache, pain or cramps in lower abdomen, and depression symptoms) for the physician's evaluation of the extent of somatic symptom intensity (a score of $0-3$ ). The modified 2011 criteria allow for clinical research without requiring an examiner interview [7].

Although these criteria have incorporated the most core symptoms of FM, it seems that a diagnostic approach of the disease which is only symptom-based has been subject to criticism from the very beginning $[8,9]$. Perceptions of physicians and patients might differ in their assessment of symptom severity due to the substantial subjectivity of criteria items [5-7]. Dropping the TPs or hyperalgesia concept as the only semi-objective finding in FM is not only the main advantage of the ACR 2010 criteria but also their greatest drawback $[8,10]$. Although many FM experts believe that TPs' examination and interpretation is difficult and may not be a valid or reliable finding in clinical settings [6], there are still some valid concerns that totally abandoning the TP concept would omit examination of the distinctive 'hyperalgesia aspect' of FM [11].

To the best of our knowledge, there has been no effort to extract and combine the best discriminative items of the existing FM diagnostic criteria. Undoubtedly, each set of diagnostic criteria has valuable properties which can be useful in correct final diagnosis. A combination of the ACR criteria (1990, 2011) seems to be able, at least partially, to address the mentioned disadvantages of each criteria. Moreover, the fibromyalgia impact questionnaire (FIQ) is another disease-specific composite measure which has some diagnostic properties for FM [12]. It has some different items in comparison to the ACR criteria, and measure different aspects of FM impact, such as functional status $[12,13]$.

The objective of the current study was to explore the ACR 1990 and 2011 classification criteria items and the FIQ components to identify the best discriminative features of FM, or "FM key features." Furthermore, we set out to provide a combined FM diagnostic (C-FM) model embracing the FM key features which can accurately identify FM patients among non-FM chronic pain disorders patients.

\section{MATERIALS AND METHODS}

\section{Study patients}

Data regarding a total of $172 \mathrm{FM}$ and 96 non-FM chronic pain (control) patients were taken from an existing FM databank [14,15]. This FM databank included patients who had been seen at six rheumatologic clinics including two teaching rheumatology clinics (Hazrat-e-Rasoul Akram General Hospital, a teaching hospital of Iran University of Medical Sciences [IUMS], and Razi General Hospital, a teaching hospital of Guilan University of Medical Sciences [GUMS]), three private rheumatology clinics and the Iranian Center for Medical Laser (ICML) affilated to Academic Center for Education, Culture and Research. We designed this study among female subjects in order to avoid if any confounding factors.

The recruitment of patients was done from September 2011 to August 2015 by three rheumatologists selected by the author (BGP, $\mathrm{AB}, \mathrm{AH}$ ) who were experienced in the diagnosis and management of chronic pain and FM. FM diagnoses in this databank were based on clinical grounds and the physician's experience, not any the ACR criteria. So, it was not a requirement of diagnosis to have satisfied the ACR 1990 or 2010 criteria. The chronic pain control group included age-matched females with a painful noninflammatory rheumatic condition such as osteoarthritis $(\mathrm{OA})$, periarthritis, or regional pain syndromes. Regional 
pain syndrome included the patients with a diagnosis of non-specific low back pain, neck pain, myofascial pain or tendinitis (such as lateral or medial epicondylitis, adhesive capsulitis, etc.) They did not have a concurrent diagnosis of FM at the time of enrollment. We divided the control group into OA (75 patients) and non-OA (21 patients) subgroups; the non-OA subgroup included patients with periarthritis or regional pain syndromes. Patients with any inflammatory rheumatic disorder, active cancer, fractures, other nonrheumatic causes for pain, and inability to read or write were excluded from the study.

This study was approved by the Ethics Committee of Guilan University of Medical Sciences. All patients signed the informed consent form.

\section{Instruments (ACR 1990 and 2011 classification criteria and FIQ)}

We compared the ACR 1990 and 2011 classification criteria items as well as FIQ components between the FM and non-FM control groups. As the widespread pain criterion (4-quadrant pain plus axial pain) in the ACR 1990 criteria is conceptually similar to the widespread pain index (WPI) in the ACR 2011 criteria, we chose 19 painful regions of WPI as the indicators of pain locations to compare FM and non-FM patients. Data regarding dolorimetric assessment of 18 defined TPs in FM and non-FM patients were collected from our databank. The pressure pain threshold was defined as the minimum force applied to induce pain. Mechanical pressure of each defined anatomical location was measured using a dolorimeter (Force Dial ${ }^{\mathrm{TM}}$ FDK20, Wagner Instrument, Greenwich, CT) and reported as $\mathrm{kg} / \mathrm{cm}^{2}$ of the skin [4]. The measured pain threshold in each site was recorded as its TP score.

The ACR 2011 classification criteria consist of two components: WPI (19 defined pain sites) and symptom severity score (6 major FM symptoms) [7,15]. We collected the results of 19 pain locations and 6 major FM symptoms evaluated in all participants, and compared FM and non-FM patients according to them. Furthermore, we added insomnia and memory disturbance (thinking or remembering problems) as dichotomous questions to our analysis. These two symptoms were part of the original ACR 2010 criteria but were later omitted in the modified 2011 criteria. It seems insomnia and memory disturbance have diagnostic importance and can be surrogate symp- toms for waking unrefreshed and cognitive symptoms, respectively [6].

The FIQ was the other tool used in this study considering its diagnostic utility in differentiating FM from nonFM $[12,16]$. The FIQ is a disease-specific composite measure composed of 10 questions [12]. We assessed all questions (20 items) in FM and non-FM patients to identify the most discriminative items between the two groups.

\section{Statistical analyses}

Descriptive statistics were used to report frequencies and scores. The means and frequency comparing FM and non-FM patients (OA and non-OA pain groups) on TPs scores, ACR 2011 components (pain locations and major FM symptoms) and FIQ items were calculated. Then, multiple regression analysis was done to identify the significant predictors of FM membership. First, the standard multiple regression analysis was done to reduce the number of variables. Actually, this stage of regression analysis identified the significant and unique predictors of group membership, thereby reducing the number of variables from 67 to 20. For the sake of preventing variable loss, we kept variables with a $P$ value of less than 0.3 in the initial model. The stepwise multiple regression analysis was then performed to order the identified variables according to their maximal statistical contribution in predicting FM versus non-FM membership. The final regression analysis model retained 8 significant variables which had best predicted FM membership. Then, the partial correlation analysis assessed the unique contribution of predictors, and the two-group discriminant analysis provided a classification table. Finally, the receiver operator characteristic (ROC) and area under the curve analyses were used to determine the sensitivity and specificity of the final model.

\section{RESULTS}

All patients were female with the mean \pm standard deviation age of $43 \pm 11$ years in FM patients and $47 \pm 11$ years in non-FM chronic pain patients. There were no statistical differences in habitancy between the FM and non-FM groups $(P=0.6)$. FM patients were different in marital status and literacy from non-FM patients $(P=0.01)$. 


\section{Tender points scores}

Nine pairs of TPS in the same anatomical location on the right and the left side were highly correlated $(R=0.811$; range: 0.74 to 0.86$)$. Therefore, to avoid multicollinearity and to reduce the number of variables, data regarding the left and right sides were averaged to form nine variables. Table 1 shows the TPs scores of FM patients, OA and nonOA chronic pain disorder patients, as well as the combined $\mathrm{OA}$ with non-OA patients (non-FM patients). The last column (FM minus non-FM) illustrates items that are most discriminatory between FM and non-FM. The highest score differences belonged to the trapezius, supraspinatus, and lateral epicondyle area $\left(>1.4 \mathrm{~kg} / \mathrm{cm}^{2}\right)$ followed by the gluteal and greater trochanter area $\left(1.2 \mathrm{~kg} / \mathrm{cm}^{2}\right)$.

\section{WPI and major FM symptoms}

Similar to TPs scores, there were acceptable correlations between the left and the right pain locations in WPI (the jaw, shoulder girdle, upper arm, lower arm, hip, upper leg, and lower leg) (mean: $R=0.619$; range: 0.51 to 0.75 ). Therefore, the left and right side pain sites were merged to reduce the number of pain locations from 19 to 12 . Table 2 shows the percentages of FM, OA, and non-OA chronic pain patients as well as $\mathrm{OA}$ and non-OA patients combined (non-FM patients) in whom ACR 2011 criteria's components (12 pain locations and major FM symptoms) were present. We merged $\mathrm{OA}$ and non-OA groups into a single group (non-FM) to increase statistical power and permit regression and discriminant analyses.

\section{FIQ scores}

The FIQ scores of FM, OA, non-OA chronic pain patients, as well as the $\mathrm{OA}$ and non-OA patients combined were calculated. In the same classification criteria sets, the calculated differences of the FIQ scores between FM and non-FM groups connoted the discriminatory properties of the FIQ items. The highest differences ( $\geq 0.7)$ between these two groups belonged to the following items: days feeling good, fatigue, and depression, all of which were worse in FM patients.

\section{Variables predicting FM versus non-FM group mem- bership}

A preliminary standard multiple logistic regression analysis was performed with the 9 pairs of TPs scores, 12 pain sites, 6 major FM symptoms, and 20 FIQ items to identify variables uniquely and statistically associated with FM and non-FM group membership. We retained the variables with a $P$ value less than 0.3 in order to prevent losing their possible contribution in the final analysis' model. This analysis identified 20 significant variables: 4 TPs (lateral epicondyle, $P=0.07$; knee, $P=0.21$; second rib, $P=0.26$; gluteal, $P=0.29$ ), 6 pain locations (neck, $P=0.003$; upper back, $P=0.004$; shoulder, $P=0.016$; hip, $P=0.01$; abdomen, $P=0.24$; lower leg, $P=0.034), 4$ major FM symptoms (insomnia, $P=0.008$; fatigue, $P=0.06$; depression, $P=0.09$; abdominal pain, $P=0.18$ ), $6 \mathrm{FIQ}$ items (physical function items [make beds, $P=0.25$; drive a car, $P=0.29$; days feeling good, $P=0.15$; work days missed, $P=0.25$;

Table 1. Tender Points Scores in Patients with Fibromyalgia, Osteoarthritis and Non-ostoearthritis

\begin{tabular}{lccccc}
\hline TPs score $\left(\mathrm{kg} / \mathrm{cm}^{2}\right)$ & FM & OA & Non-OA & $\begin{array}{c}\text { OA and non-OA } \\
\text { (non-FM) }\end{array}$ & $\begin{array}{c}\text { Difference in TP score } \\
\text { between FM and non-FM }\end{array}$ \\
\hline Low cervical & $1.3 \pm 0.4$ & $1.7 \pm 0.4$ & $1.7 \pm 0.5$ & $1.7 \pm 0.4$ & $-0.4 \pm-0.1$ \\
Second rib & $2.0 \pm 0.8$ & $3.2 \pm 0.8$ & $2.9 \pm 0.5$ & $3.1 \pm 0.7$ & $-1.1 \pm-0.3$ \\
Lateral epicondyle & $2.4 \pm 0.9$ & $4.0 \pm 0.9$ & $3.9 \pm 0.7$ & $4.0 \pm 0.8$ & $-1.4 \pm 0.2$ \\
Knee & $2.5 \pm 0.9$ & $3.2 \pm 1.2$ & $3.7 \pm 1.0$ & $3.3 \pm 1.1$ & $-0.8 \pm-0.1$ \\
Occiput & $2.0 \pm 0.8$ & $3.0 \pm 0.8$ & $3.0 \pm 0.6$ & $3.0 \pm 0.7$ & $-1.0 \pm 0.1$ \\
Trapezius & $2.3 \pm 0.9$ & $3.8 \pm 0.9$ & $3.8 \pm 0.9$ & $3.8 \pm 0.9$ & $-1.5 \pm-0.0$ \\
Supraspinatus & $2.6 \pm 0.9$ & $4.1 \pm 0.8$ & $4.1 \pm 1.0$ & $4.1 \pm 0.8$ & $-1.4 \pm-0.0$ \\
Gluteal & $3.1 \pm 0.9$ & $4.4 \pm 0.6$ & $4.3 \pm 0.9$ & $4.4 \pm 0.7$ & $-1.3 \pm-0.3$ \\
Greater trochanter & $3.1 \pm 0.8$ & $4.4 \pm 0.8$ & $4.4 \pm 0.9$ & $4.4 \pm 0.8$ & $-1.2 \pm-0.1$ \\
\hline
\end{tabular}

Values are presented as mean \pm SD. $P$-values in all TP score differences between FM and non-FM were $P<0.01$. TP: tender point, FM: fibromyalgia, OA: osteoarthritis. 
Table 2. American College of Rheumatology 2011 Classification Criteria Components' Measures

\begin{tabular}{|c|c|c|c|c|c|}
\hline Variable & FM & $\mathrm{OA}$ & Non-OA & $\begin{array}{l}\text { OA and non-OA } \\
\text { (non-FM) }\end{array}$ & $\begin{array}{l}\text { Difference between } \\
\text { FM and non-FM }\end{array}$ \\
\hline \multicolumn{6}{|l|}{ Pain location } \\
\hline Neck & 77.2 & 16.0 & 23.8 & 17.7 & 59.5 \\
\hline Shoulder & 79.5 & 18.7 & 23.8 & 19.8 & 59.7 \\
\hline Upper arm & 51.5 & 6.7 & 19.0 & 9.4 & 42.1 \\
\hline Lower arm & 48.0 & 9.3 & 38.1 & 15.6 & 32.4 \\
\hline Chest & 29.2 & 4 & 14.3 & 6.3 & 22.9 \\
\hline Abdomen & 18.7 & 1.3 & 0 & 1.0 & 17.7 \\
\hline Upper leg & 39.2 & 9.3 & 19.0 & 11.5 & 27.7 \\
\hline Lower leg & 62.6 & 64.0 & 47.6 & 60.4 & 2.2 \\
\hline Jaw & 12.3 & 0 & 0 & 0 & 12.3 \\
\hline Upper back & 58.5 & 6.7 & 14.3 & 8.3 & 50.2 \\
\hline Low back & 59.1 & 16.0 & 14.3 & 15.6 & 43.5 \\
\hline Hip & 52.6 & 8.0 & 4.8 & 7.3 & 45.3 \\
\hline \multicolumn{6}{|l|}{ Major FM symptom } \\
\hline Fatigue & $2.0 \pm 0.8$ & $0.9 \pm 0.9$ & $0.8 \pm 0.9$ & $0.9 \pm 0.9$ & $1.1 \pm-0.1$ \\
\hline Waking unrefreshed & $1.9 \pm 0.8$ & $0.8 \pm 0.9$ & $0.9 \pm 1.0$ & $0.8 \pm 0.9$ & $1.1 \pm-0.1$ \\
\hline Cognitive symptom & $1.3 \pm 0.8$ & $0.6 \pm 0.7$ & $0.5 \pm 0.5$ & $0.5 \pm 0.7$ & $0.8 \pm 0.1$ \\
\hline Memory disturbance & 81.3 & 46.6 & 52.4 & 47.9 & 33.4 \\
\hline Insomnia & 82.6 & 40.5 & 19.0 & 35.0 & 63.6 \\
\hline Abdominal pain & 55.8 & 28.0 & 42.9 & 31.3 & 12.9 \\
\hline Headache & 82.6 & 45.3 & 33.3 & 42.7 & 49.3 \\
\hline Depression & 87.8 & 40.5 & 33.3 & 38.9 & 54.5 \\
\hline
\end{tabular}

Values are presented as percentage or mean \pm SD. $P$-values in all percentages differences between FM and non-FM were $P<0.01$. ACR: American College of Rheumatology, FM: fibromyalgia, OA: osteoarthritis.

fatigue, $P=0.02$; depression, $P=0.01$.

These 20 variables were then entered into a stepwise multiple logistic regression analysis (Table 3) to identify variables that best differentiated the FM group from nonFM group. The analysis identified and ordered the 8 best discriminative variables according to their maximal statistical contribution in predicting FM versus non-FM membership. This model with 8 predictors accounted for $64.8 \%$ of the variance associated with FM group membership (Table 3, column 3, bottom row). The most important predictors of group membership in order of magnitude were as follows (variance accounted for and FM vs. non- FM differences): lateral epicondyle TP $\left(36.9 \% ; 2.4 \mathrm{~kg} / \mathrm{cm}^{2}\right.$ vs. 4.0 $\mathrm{kg} / \mathrm{cm}^{2}$ ), neck pain (14.5\%; $77.2 \%$ vs. $\left.17.7 \%\right)$, fatigue (4.7\%; $2.0 \%$ vs. $0.9 \%$ ), insomnia (3\%; $82.6 \%$ vs. $35.0 \%$ ), upper back pain $(2.2 \% ; 58.5 \%$ vs. $8.3 \%)$, shoulder pain $(1.5 \%$; $79.5 \%$ vs. $19.8 \%)$ and gluteal TP $\left(1.2 \% ; 3.1 \mathrm{~kg} / \mathrm{cm}^{2}\right.$ vs. 4.4 $\mathrm{kg} / \mathrm{cm}^{2}$ ), FIQ fatigue (0.9\%; 6.2 vs. 5.3).

Table 4 shows the unique contribution (partial correlations) of each variable when other variables were controlled.
The lateral epicondyle TP which was the first variable to be retained in the stepwise regression analysis had a strong, unique, and statistically significant partial correlation $(R=0.220)$. Notably, insomnia and fatigue demonstrated the strongest unique partial correlations $(R=0.271$ and 0.224 , respectively) and also substantial zero-order correlations ( $R=0.471$ and 0.531 , respectively). The FIQ fatigue item, which had a low FM/non-FM score differences, also had low zero-order and partial correlations (Table 4).

Finally, discriminant function analysis was done to classify FM and non-FM individuals according to the final eight-variable list. Using this model, which we named the "combined FM model or C-FM model," 91.4\% of patients were correctly classified (Table 5). ROC curve analysis determined the best cutoff point for the C-FM model. We found high sensitivity and specificity for this model (91.9\% and $91.7 \%$, respectively). 


\section{DISCUSSION}

The analysis of 67 items derived from the ACR 1990 and 2011 FM classification criteria and the FIQ identified the best constellation of key features of FM which can be useful in FM diagnosis. We introduced the $\mathrm{C}-\mathrm{FM}$ model which

Table 3. Stepwise Multiple Regression Analysis Showing 8 Predictors Ranked in Order of Magnitude in Predicting Group Membership (FM or Non-FM)

\begin{tabular}{|c|c|c|c|c|}
\hline Predictor & Multiple $R$ & Multiple $R^{2}$ & $R^{2}$ change $^{a}$ & $P$ value \\
\hline $\begin{array}{l}\text { Lateral epicondyle } \\
\text { TP mean score }\end{array}$ & 0.607 & 0.369 & 0.369 & $<0.01$ \\
\hline Neck pain & 0.717 & 0.514 & 0.145 & $<0.01$ \\
\hline $\begin{array}{l}\text { ACR } 2011 \text {-fatigue } \\
\text { score }\end{array}$ & 0.749 & 0.560 & 0.047 & $<0.01$ \\
\hline Insomnia & 0.768 & 0.590 & 0.030 & $<0.01$ \\
\hline Upper back pain & 0.782 & 0.612 & 0.022 & $<0.01$ \\
\hline Shoulder pain & 0.792 & 0.627 & 0.015 & $<0.01$ \\
\hline $\begin{array}{l}\text { Gluteal TP mean } \\
\text { score }\end{array}$ & 0.799 & 0.638 & 0.012 & $<0.01$ \\
\hline FIQ-fatigue score & $0.805^{b}$ & $0.648^{c}$ & 0.009 & $<0.01$ \\
\hline
\end{tabular}

The forward stepwise regression analysis used 8 predictors which combined to produce a multiple $R=0.805$ (last row, column 2). This accounted for $64.8 \%$ of variance associated with group membership (column 3).

FM: fibromyalgia, TP: tender point, ACR: American College of Rheumatology, FIQ: fibromyalgia impact questionnaire.

${ }^{a} R^{2}$ change represents prediction percentage of each variable in FM membership.

${ }^{b}$ The forward stepwise regression analysis used 8 predictors which combined to produce a multiple $R=0.805$.

'This accounted for $64.8 \%$ of variance associated with group membership. provides a practical diagnostic tool to accurately differentiate FM among chronic pain disorders. Some valuable features from both sets of ACR criteria remained as the important discriminative features in the final model, but the FIQ components showed poor discriminatory properties in our analysis.

Given the fact that all the existing diagnostic classification criteria for FM have been faced with some challenges and impose some burden on physicians [5,17], development of a combined model in order to address disadvantages of previous criteria seems essential. Exploring 67 items taken from the 3 criteria sets to find the best discriminatory features of FM was the first attempt in this line. From a conceptual perspective, the combination of two TPs (lateral epicondyles and gluteal) and axial pain

Table 5. Correct Classification as Predicted by C-FM Model Using 8 Key Features

\begin{tabular}{lcrr}
\hline \multirow{2}{*}{ Variable } & \multicolumn{2}{c}{ Predicted group membership (\%) } & Total \\
\cline { 2 - 3 } & Non-FM & \multicolumn{1}{c}{ FM } & \\
\hline Original & & & \\
$\quad$ Non-FM & $86(89.6)$ & $10(10.4)$ & 96 \\
FM & $13(7.6)$ & $159(92.4)$ & 172 \\
Cross-validated ${ }^{b}$ & & & \\
Non-FM & $83(86.5)$ & $13(13.5)$ & 96 \\
FM & $14(8.1)$ & $158(91.9)$ & 172 \\
\hline
\end{tabular}

FM: fibromyalgia, C-FM: combined FM.

${ }^{a} 91.4 \%$ of original grouped cases correctly classified. ${ }^{b}$ Cross validation is done only for those cases in the analysis. In cross validation, each case is classified by the functions derived from all cases other than that case. $89.9 \%$ of cross-validated grouped cases correctly classified.

Table 4. Zero Order (Pearson's R) and Partial Correlations of Predictors in the Final Model

\begin{tabular}{lcccc}
\hline \multicolumn{1}{c}{ Predictor } & Pearson's $R$ & Partial $R$ & $P$ value & Odd ratio (95\% Cl) \\
\hline Lateral epicondyle TP mean score & -0.607 & -0.220 & $<0.01$ & $0.46(0.2-0.8)$ \\
Neck pain & 0.574 & 0.217 & $<0.01$ & $3.1(0.9-10.4)$ \\
ACR 2011-fatigue score & 0.530 & 0.224 & $<0.01$ & $3.0(1.5-5.9)$ \\
Insomnia & 0.471 & 0.271 & $<0.01$ & $9.9(2.9-33.8)$ \\
Upper back pain & 0.490 & 0.201 & $<0.01$ & $3.4(0.9-12.4)$ \\
Shoulder pain & 0.580 & 0.181 & $<0.01$ & $2.2(0.6-7.6)$ \\
Gluteal TP mean score & -0.584 & -0.172 & $<0.01$ & $0.3(1.0-0.6)$ \\
FlQ-fatigue score & 0.151 & 0.16 & $<0.01$ & $1.1(0.9-1.3)$
\end{tabular}

Minus correlation indicate that FM patients have lower scores on predictor variable. All Pearson's correlations are significant. $\mathrm{Cl}$ : confidence interval, TP: tender point, ACR: American College of Rheumatology, FIQ: fibromyalgia impact questionnaire. 
(neck and upper back pain), along with fatigue and insomnia produced a substantial variance in group membership (65\%), with a correct classification rate of $91.4 \%$. This study shows that all these predictors together can be considered as a valid model for the diagnosis of FM. Furthermore, unique contributions of these predictors were approximately equal in the classification of FM and non-FM patients (Table 4). According to this finding, it seems that the TPs, axial pain, fatigue, and insomnia which derived from the ACR 1990 and 2011 criteria play equal substantial roles when they are independently considered to differentiate FM from non-FM patients. On the other hand, the FIQ components showed poor discriminatory properties in all the analyses.

Lateral epicondyle TP, the first predictor in the C-FM model, was responsible for $36.9 \%$ of variance (Table 3 ). This indicates a unique role for this key feature in separating FM from other chronic pain disorders. This finding is contrary to the logics of elimination of TPs from the ACR 2010 classification criteria which were the inherently arbitrary nature and low validation of TPs examination [6]. Although the ACR 2010 criteria study showed that muscle tenderness was one of the most important variables classifying cases and non-cases of FM, tenderness was not used in the final formulation of this criteria $[6,10]$. It is worthwhile to note that TPs have traditionally been thought as a clinical indicator of lowered pain thresholds [18]. Further, centrally mediated augmented pain and sensory processes have firmly been established to play a pivotal role in the hyperalgesia of FM patients [11,18]. Such a pathophysiological concept implicates that TPs could be used as an important and intuitive pointer of FM diagnosis [8]. So, it seems that TPs as a unique feature of hyperalgesia in FM can still be saved for more valid diagnosis.

Furthermore, our study showed that the examination of two TPs (lateral epicondyles and gluteal) would efficiently differentiate FM from non-FM, especially if TPs are considered intimately with other major symptoms such as axial pain and fatigue. Undoubtedly, using fewer but more valid discriminatory TPs (examination of only 2 instead of 9 TP pairs in the ACR 1990 criteria) can facilitate FM diagnosis by non-rheumatologic physicians and resolves the previous problems described with the ACR 1990 classification criteria. This is in line with previous studies demonstrating that some TPs in fewer numbers might have more diagnostic value in differentiating FM from non-FM patients $[14,19,20]$.

Another aspect of $\mathrm{C}-\mathrm{FM}$ model is four components of the ACR 2011 criteria which have remained in this model. Interestingly, high axial pain including neck and high back pain showed substantial variance $(14.5 \%$ and $2 \%$, respectively) in the group membership. It seems that axial pain plays more important role in discrimination of FM from non-FM rather than other pain locations [21]. These predictors along with fatigue and insomnia accounted for important contribution in FM membership.

Fatigue, a cardinal symptom in FM, remained in two manners in the final model, either as an ACR 2011 criteria component or as a FIQ item. Actually, fatigue has been shown to be the second most reported symptom in fibromyalgia [7,22]. Our findings are in agreement with such a general and consistent pattern of fatigue perception by FM patients. In fact, FIQ's fatigue was the only item of FIQ in the current study that showed to have a discriminatory property. On the other hand, other FIQ components played a minimal role in differentiating FM from non-FM patients.

The surprising notion was omission of other FM symptoms such as waking unrefreshed, cognitive and the somatic symptoms from the C-FM model, indicating the low discriminatory power of these variables. They constitute a pivotal portion of the ACR 2011 criteria, and the general knowledge supports their role in FM diagnosis. In our study, however, insomnia showed a better discriminatory property than waking unrefreshed. The reason might be due to the difficult evaluation of waking unrefreshed rather than insomnia probably because the higher subjectivity nature of the former. Nonetheless, waking unrefreshed might still be considered as a surrogate marker for insomnia. Furthermore, cognitive dysfunction as another major component of the ACR 2011 classification criteria had a low discriminatory ability in our study. It concurs with the Tesio et al. [23] study in which different neuropsychological tests showed minor differences between FM and non-FM patients regarding cognitive function. Based on our study and previous literature $[19,23]$ it seems that accepting cognitive function as a FM discriminatory feature still needs further investigation.

To the best of our knowledge, this is the first study attempting to explore and combine the discriminative features of both 1990 and 2011 versions of the ACR classification criteria. It seems that both classification criteria 
have valuable key features whose combination can accurately detect FM. Re-inclusion of an examination of fewer, but the most discriminative TPs, and decreased subjectivity in evaluation of FM are the strengths of the C-FM model. Although it is desirable for FM patients to be diagnosed and cared for by rheumatologists, in reality this is not always possible nor is necessary for most patients $[24,25]$. So, the simplicity and holistic nature of the C-FM model can facilitate diagnosis of FM patients in a realistic manner by physicians.

Our study had some limitations. Only female patients were evaluated in this study and therefore the results of the study cannot be generalized to male patients with FM. Moreover, in this study, diagnosis of fibromyalgia was made based on expert opinion and clinical grounds, not on the ACR criteria. Although use of expert diagnosis as the gold standard for assessment of the discriminative ability of the existing ACR criteria items in FM diagnosis was inevitable, it remained a weak point of our study. Furthermore, we are not aware of the validity of the C- FM model in other populations and care settings. We think that the next step would be evaluation of the C-FM model's validity in primary care setting as well as in patients with other rheumatic conditions. Finally, although the number of $\mathrm{pa}^{-}$ tients included in this study was relatively good, further studies with larger populations still need to be done to better evaluate the diagnostic capabilities of the C-FM model.

In summary, the $\mathrm{C}-\mathrm{FM}$ model can accurately detect FM patients among other pain disorders by using fewer but more discriminative TPs along with high axial pain, fatigue, and insomnia. Using fewer discriminatory TPs can conserve the intuitive and unique hyperalgesia feature of FM. The combination of 8 key features derived from both the 1990 and 2011 ACR criteria forming the C-FM model showed substantial discriminatory properties with a $91.4 \%$ correct classification rate. Re-inclusion of TPs and decreased subjectivity along with retaining FM's main symptoms in the $\mathrm{C}-\mathrm{FM}$ model can be considered a unique feature of this model.

\section{CONFLICT OF INTEREST}

No potential conflict of interest relevant to this article was reported.

\section{REFERENCES}

1. Borchers AT, Gershwin ME. Fibromyalgia: a critical and comprehensive review. Clin Rev Allergy Immunol 2015; 49: 100-51.

2. Mease PJ, Arnold LM, Crofford LJ, Williams DA, Russell IJ, Humphrey $L$, et al. Identifying the clinical domains of fibromyalgia: contributions from clinician and patient Delphi exercises. Arthritis Rheum 2008; 59: 952-60.

3. Wolfe F, Walitt B. Culture, science and the changing nature of fibromyalgia. Nat Rev Rheumatol 2013; 9: 751-5.

4. Wolfe F, Smythe HA, Yunus MB, Bennett RM, Bombardier C, Goldenberg DL, et al. The American College of Rheumatology 1990 criteria for the classification of fibromyalgia. Report of the Multicenter Criteria Committee. Arthritis Rheum 1990; 33: $160-72$.

5. Wolfe F. Editorial: the status of fibromyalgia criteria. Arthritis Rheumatol 2015; 67: 330-3.

6. Wolfe F, Clauw DJ, Fitzcharles MA, Goldenberg DL, Katz RS, Mease $\mathrm{P}$, et al. The American College of Rheumatology preliminary diagnostic criteria for fibromyalgia and measurement of symptom severity. Arthritis Care Res (Hoboken) 2010; 62: 600-10.

7. Wolfe F, Clauw DJ, Fitzcharles MA, Goldenberg DL, Häuser W. Katz RS, et al. Fibromyalgia criteria and severity scales for clinical and epidemiological studies: a modification of the ACR Preliminary Diagnostic Criteria for Fibromyalgia. J Rheumatol 2011; 38: 1113-22.

8. Egloff N, von Känel R, Müller V, Egle UT, Kokinogenis G, Lederbogen $\mathrm{S}$, et al. Implications of proposed fibromyalgia criteria across other functional pain syndromes. Scand J Rheumatol 2015; 44: 416-24.

9. Smythe HA. Unhelpful criteria sets for "diagnosis" and "assessment of severity" of fibromyalgia. J Rheumatol 2011; 38: $975-8$.

10. Friend R, Bennett RM. A critical examination of the polysymptomatic distress scale construct as a symptom severity questionnaire. J Rheumatol 2015; 42: 1364-7.

11. Desmeules JA, Cedraschi C, Rapiti E, Baumgartner E, Finckh A, Cohen P, et al. Neurophysiologic evidence for a central sensitization in patients with fibromyalgia. Arthritis Rheum 2003; 48: 1420-9.

12. Bennett R. The Fibromyalgia Impact Questionnaire $(F \mid Q)$ : a review of its development, current version, operating characteristics and uses. Clin Exp Rheumatol 2005; 23(5 Suppl 39): S154-62.

13. Bennett RM, Friend R, Marcus D, Bernstein C, Han BK, Yachoui R, et al. Criteria for the diagnosis of fibromyalgia: validation of the modified 2010 preliminary American College of Rheumatology criteria and the development of alternative criteria. Arthritis Care Res (Hoboken) 2014; 66: 1364-73.

14. Ghavidel-Parsa B, Bidari A, Maafi AA, Hassankhani A, 
Hajiabbasi A, Montazeri A, et al. The impact of fibromyalgia on health status according to the types, demographic background and pain index. Clin Exp Rheumatol 2016; 34(2 Suppl 96): S134-9.

15. Bidari A, Ghavidel-Parsa B, Amir Maafi A, Montazeri A, Ghalehbaghi B, Hassankhani A, et al. Validation of fibromyalgia survey questionnaire and polysymptomatic distress scale in a Persian population. Rheumatol Int 2015; 35: 2013-9.

16. Bidari A, Hassanzadeh M, Mohabat MF, Talachian E, Khoei EM. Validation of a Persian version of the Fibromyalgia Impact Questionnaire (FIQ-P). Rheumatol Int 2014; 34: 181-9.

17. Wolfe F, Clauw DJ, Fitzcharles MA, Goldenberg DL, Häuser W, Katz RL, et al. 2016 Revisions to the 2010/2011 fibromyalgia diagnostic criteria. Semin Arthritis Rheum 2016; 46 : 319-29.

18. Schmidt-Wilcke T, Clauw DJ. Fibromyalgia: from pathophysiology to therapy. Nat Rev Rheumatol 2011; 7: 518-27.

19. Segura-Jiménez V, Álvarez-Gallardo IC, Carbonell-Baeza A, Aparicio VA, Ortega FB, Casimiro AJ, et al. Fibromyalgia has a larger impact on physical health than on psychological health, yet both are markedly affected: the al-Ándalus project. Semin Arthritis Rheum 2015; 44: 563-70.

20. Tastekin N, Uzunca K, Sut N, Birtane M, Mercimek OB.
Discriminative value of tender points in fibromyalgia syndrome. Pain Med 2010; 11: 466-71.

21. Friend R, Bennett RM. Distinguishing fibromyalgia from rheumatoid arthritis and systemic lupus in clinical questionnaires: an analysis of the revised Fibromyalgia Impact Questionnaire (FIQR) and its variant, the Symptom Impact Questionnaire (SIQR), along with pain locations. Arthritis Res Ther 2011; 13: R58.

22. Silverman SL, Harnett J, Zlateva G, Mardekian J. Identifying fibromyalgia-associated symptoms and conditions from a clinical perspective: a step toward evaluating healthcare resource utilization in fibromyalgia. Pain Pract 2010; 10: 520-9.

23. Tesio V, Torta DM, Colonna F, Leombruni P, Ghiggia A, Fusaro E, et al. Are fibromyalgia patients cognitively impaired? Objective and subjective neuropsychological evidence. Arthritis Care Res (Hoboken) 2015; 67: 143-50.

24. Fitzcharles MA, Ste-Marie PA, Goldenberg DL, Pereira JX, Abbey S, Choinière M, et al. 2012 Canadian Guidelines for the diagnosis and management of fibromyalgia syndrome: executive summary. Pain Res Manag 2013; 18: 119-26.

25. Ghavidel-Parsa B, Amir Maafi A, Aarabi Y, Haghdoost A, Khojamli M, Montazeri A, et al. Correlation of invalidation with symptom severity and health status in fibromyalgia. Rheumatology (Oxford) 2015; 54: 482-6. 